\title{
Convergence in Measure of Logarithmic Means of Quadratical Partial Sums of Double Walsh-Kaczmarz-Fourier Series
}

\author{
Ushangi Goginava ${ }^{1}$ and Károly $\mathbf{N a g y}^{2}$ \\ ${ }^{1}$ Institute of Mathematics, Faculty of Exact and Natural Sciences, Tbilisi State University, \\ Chavchavadze Street 1, 0128 Tbilisi, Georgia \\ ${ }^{2}$ Institute of Mathematics and Computer Sciences, College of Nyíregyháza, \\ P.O. Box 166, Nyíregyháza 4400, Hungary
}

Correspondence should be addressed to Károly Nagy, nkaroly@nyf.hu

Received 31 January 2011; Accepted 3 April 2011

Academic Editor: Anna Kaminska

Copyright (c) 2012 U. Goginava and K. Nagy. This is an open access article distributed under the Creative Commons Attribution License, which permits unrestricted use, distribution, and reproduction in any medium, provided the original work is properly cited.

The main aim of this paper is to prove that the logarithmic means of quadratical partial sums of the double Walsh-Kaczmarz series does not improve the convergence in measure. In other words, we prove that for any Orlicz space, which is not a subspace of $L \log ^{+} L\left(I^{2}\right)$, the set of the functions the logarithmic means of quadratical partial sums of the double Walsh-Kaczmarz series of which converge in measure is of first Baire category.

\section{Definitions and Notations}

We denote by $L^{0}=L^{0}\left(I^{2}\right)$ the Lebesgue space of functions that are measurable and finite almost everywhere on $I^{2}=[0,1) \times[0,1)$. mes $(A)$ is the Lebesgue measure of the set $A \subset I^{2}$. The constants appearing in this paper are denoted by $c$.

Let $L_{\Phi}=L_{\Phi}\left(I^{2}\right)$ be the Orlicz space [1] generated by Young's function $\Phi$; that is, $\Phi$ is convex, continuous, even function such that $\Phi(0)=0$ and

$$
\lim _{u \rightarrow+\infty} \frac{\Phi(u)}{u}=+\infty, \quad \lim _{u \rightarrow 0} \frac{\Phi(u)}{u}=0 .
$$

This space is endowed with the norm

$$
\|f\|_{L_{\Phi}\left(I^{2}\right)}=\inf \left\{k>0: \int_{I^{2}} \Phi\left(\frac{|f(x, y)|}{k}\right) d x d y \leq 1\right\} .
$$


In particular case, if $\Phi(u)=u \ln (1+u), u>0$, then the corresponding space is denoted by $L \log ^{+} L\left(I^{2}\right)$.

We denote the set of nonnegative integers by $\mathbf{N}$. By a dyadic interval in $I:=[0,1)$ we mean one of the form $\left[p / 2^{n},(p+1) / 2^{n}\right)$ for some $p \in \mathbf{N}, 0 \leq p<2^{n}$. Given $n \in \mathbf{N}$ and $x \in[0,1)$, let $I_{n}(x)$ denote the dyadic interval of length $2^{-n}$ which contains the point $x$.

Every point $x \in I$ can be written in the following form:

$$
x=\sum_{k=0}^{\infty} \frac{x_{k}}{2^{k+1}}=:\left(x_{0}, x_{1}, \ldots, x_{n}, \ldots\right), \quad x_{k} \in\{0,1\}
$$

In that case when there are two different forms we choose the one for which $\lim _{k \rightarrow \infty} x_{k}=0$. We use the notation

$$
e_{j}:=\frac{1}{2^{j+1}}=\left(0, \ldots, 0, x_{j}=1,0, \ldots\right)
$$

It is well-known that $[2,3]$

$$
I_{n}\left(x_{0}, \ldots, x_{n-1}\right):=I_{n}(x)=\left[\frac{p}{2^{n}}, \frac{p+1}{2^{n}}\right)
$$

where $p=\sum_{j=0}^{n-1} x_{j} 2^{n-1-j}$.

Let $r_{0}(x)$ be a function defined by

$$
r_{0}(x)=\left\{\begin{array}{ll}
1, & \text { if } x \in\left[0, \frac{1}{2}\right), \\
-1, & \text { if } x \in\left[\frac{1}{2}, 1\right),
\end{array} \quad r_{0}(x+1)=r_{0}(x)\right.
$$

The Rademacher functions are defined by

$$
r_{n}(x)=r_{0}\left(2^{n} x\right), \quad n \geq 0, x \in[0,1)
$$

Let $w_{0}, w_{1}, \ldots$ represent the Walsh functions, that is, $w_{0}(x)=1$, and if $n=\sum_{i=0}^{\infty} n_{i} 2^{i}$ is a positive integer with $n_{i} \in\{0,1\}$, then

$$
w_{n}(x):=\prod_{i=0}^{\infty}\left(r_{i}(x)\right)^{n_{i}} .
$$

The order of the $n$ is denoted by $|n|:=\max \left\{j \in \mathbf{N}: n_{j} \neq 0\right\}$.

The Walsh-Kaczmarz functions are given by $\kappa_{0}:=1$, and for $n \geq 1$

$$
\kappa_{n}(x):=r_{|n|}(x) \prod_{k=0}^{|n|-1}\left(r_{|n|-1-k}(x)\right)^{n_{k}}
$$


For $A \in \mathbf{N}$ and $x \in I$, Skvorcov [4] defined a transformation $\tau_{A}: I \rightarrow I$ by

$$
\tau_{A}(x):=\sum_{k=0}^{A-1} x_{A-k-1} 2^{-(k+1)}+\sum_{j=A}^{\infty} x_{j} 2^{-(j+1)}
$$

By the definition of $\tau_{A}$, we have (see [4])

$$
\kappa_{n}(x)=r_{|n|}(x) w_{n-2^{|n|}}\left(\tau_{|n|}(x)\right) \quad(n \in \mathbf{N}, x \in I) .
$$

The Dirichlet kernels are defined by

$$
D_{n}^{\alpha}(x):=\sum_{k=0}^{n-1} \alpha_{k}(x)
$$

where $\alpha_{k}=w_{k}$ for all $k \in \mathbf{N}$ or $\kappa_{k}$ for all $k \in \mathbf{N}$.

It is well-known that $[2,4]$

$$
\begin{gathered}
D_{n}^{\kappa}(x)=D_{2^{|n|}}(x)+w_{2^{|n|}}(x) D_{n-2^{|n|}}^{w}\left(\tau_{|n|}(x)\right), \\
D_{2^{n}}(x):=D_{2^{n}}^{w}(x)=D_{2^{n}}^{\kappa}(x)= \begin{cases}2^{n}, & \text { if } x \in\left[0, \frac{1}{2^{n}}\right), \\
0, & \text { if } x \in\left[\frac{1}{2^{n}}, 1\right) .\end{cases}
\end{gathered}
$$

The $n$th Fejér means of the Walsh-(Kaczmarz-)Fourier series of function $f$ are given by

$$
\sigma_{n}^{\alpha}(f, x):=\frac{1}{n} \sum_{j=1}^{n} S_{j}^{\alpha}(f, x)
$$

where

$$
S_{n}^{\alpha}(f, x)=\sum_{k=0}^{n-1} \widehat{f}^{\alpha}(k) \alpha_{k}(x)
$$

is the $n$th partial sum of the Fourier series and $\hat{f}^{\alpha}(n):=\int_{I} f \alpha_{n}(n \in \mathbf{N})$ is said to be the $n$th Walsh-(Kaczmarz-)Fourier coefficient of the function $f$.

The Nörlund logarithmic (simply we say logarithmic) means and kernels of onedimensional Walsh-(Kaczmarz-)Fourier series are defined as follows:

$$
t_{n}^{\alpha}(f, x)=\frac{1}{l_{n}} \sum_{k=1}^{n-1} \frac{S_{k}^{\alpha}(f, x)}{n-k}, \quad F_{n}^{\alpha}(t)=\frac{1}{l_{n}} \sum_{k=1}^{n-1} \frac{D_{k}^{\alpha}(t)}{n-k}
$$

where $l_{n}=\sum_{k=1}^{n-1} 1 / k$. 
The Kronecker product $\left(\alpha_{m, n}: n, m \in \mathbf{N}\right)$ of two Walsh(-Kaczmarz) systems is said to be the two-dimensional Walsh(-Kaczmarz) system. That is,

$$
\alpha_{m, n}(x, y)=\alpha_{m}(x) \alpha_{n}(y)
$$

If $f \in L\left(I^{2}\right)$, then the number $\widehat{f}^{\alpha}(m, n):=\int_{I^{2}} f \alpha_{m, n}(n, m \in \mathbf{N})$ is said to be the $(m, n)$ th Walsh-(Kaczmarz-)Fourier coefficient of $f$. The rectangular partial sums of double Fourier series with respect to both system are defined by

$$
S_{m, n}^{\alpha}(f, x, y)=\sum_{i=0}^{m-1} \sum_{j=0}^{n-1} \widehat{f}^{\alpha}(i, j) \alpha_{i}(x) \alpha_{j}(y)
$$

The logarithmic means and kernels of quadratical partial sums of double Walsh(Kaczmarz)-Fourier series are given by

$$
\mathbf{t}_{n}^{\alpha}(f, x, y)=\frac{1}{l_{n}} \sum_{k=1}^{n-1} \frac{S_{k, k}^{\alpha}(f, x, y)}{n-k}, \quad \mathbf{F}_{n}^{\alpha}(x, y)=\frac{1}{l_{n}} \sum_{k=1}^{n-1} \frac{D_{k}^{\alpha}(x) D_{k}^{\alpha}(y)}{n-k}
$$

It is evident that

$$
\mathbf{t}_{n}^{\alpha}(f, x, y)-f(x, y)=\int_{I^{2}}[f(x \oplus t, y \oplus s)-f(x, y)] \mathbf{F}_{n}^{\alpha}(t, s) d t d s
$$

where $\oplus$ denotes the dyadic addition [3]. The $n$th Marcinkiewicz kernels are given by

$$
\mathcal{K}_{n}^{\alpha}(x, y):=\frac{1}{n} \sum_{k=1}^{n} D_{k}^{\alpha}(x) D_{k}^{\alpha}(y)
$$

In 1948 Šneřder [5] showed that the inequality

$$
\limsup _{n \rightarrow \infty} \frac{D_{n}^{\kappa}(x)}{\log n} \geq C>0
$$

holds a.e. for the Walsh-Kaczmarz Dirichlet kernel. This inequality shows that the behavior of the Walsh-Kaczmarz system is worse than the behavior of the Walsh system in the Paley enumeration. This "spreadness" property of the kernel makes it easier to construct examples of divergent Fourier series [6]. On the other hand, Schipp [7] and Young [8] in 1974 proved that the Walsh-Kaczmarz system is a convergence system. Skvorcov in 1981 [4] showed that the Fejér means with respect to the Walsh-Kaczmarz system converge uniformly to $f$ for any continuous functions $f$. For any integrable functions, Gát [9] proved that the Fejér means with respect to the Walsh-Kaczmarz system converge a.e. to the function. Recently, Gát's result was generalized by Simon $[10,11]$. The a.e. convergence of the Walsh-KaczmarzMarcinkiewicz means of integrable functions was discussed by the second author [12]. 
The partial sums $S_{n}^{w}(f)$ of the Walsh-Fourier series of a function $f \in L(I), I=[0,1)$ converge in measure on $I$ [2]. The condition $f \in L \log ^{+} L\left(I^{2}\right)$ provides convergence in measure on $I^{2}$ of the rectangular partial sums $S_{n, m}^{w}(f)$ of double Walsh-Fourier series [13]. The first example of a function from classes wider than $L \log ^{+} L\left(I^{2}\right)$ with $S_{n, n}^{w}(f)$ divergent in measure on $I^{2}$ was obtained in $[14,15]$. Moreover, in [16] Tkebuchava proved that in each Orlicz space wider than $L \log ^{+} L\left(I^{2}\right)$ the set of functions where quadratic Walsh-Fourier sums converge in measure on $I^{2}$ is of first Baire category (see Goginava [17] for Walsh-Kaczmarz-Fourier series). In the paper [18] it was showed that the Nörlund logarithmic means $t_{n}^{\kappa} f$ of onedimensional Walsh-Kaczmarz-Fourier series are of weak type $(1,1)$, and this fact implies that $t_{n}^{\kappa} f$ converge in measure on $I$ for all functions $f \in L(I)$ and $t_{n, m}^{\kappa} f$ converge in measure on $I^{2}$ for all functions $f \in L \log ^{+} L\left(I^{2}\right)$.

At last, we note that the Walsh-Nörlund logarithmic means are closer to the partial sums than to the classical logarithmic means or the Fejér means. Namely, it was proved that there exists a function in a certain class of functions and a set with positive measure, such that the Walsh-Nörlund logarithmic means of the function diverge on the set [19].

For results with respect to logarithmic means of cubical and rectangular partial sums of two-dimensional Walsh-Fourier series, see [17, 19-22].

In the present paper we investigate convergence in measure of logarithmic means of quadratical partial sums

$$
\frac{1}{l_{n}} \sum_{i=1}^{n-1} \frac{S_{i, i}(f, x, y)}{n-i}
$$

of double Walsh-Kaczmarz series and prove Theorem 2.1 that is, for any Orlicz space, which is not a subspace of $L \log ^{+} L\left(I^{2}\right)$, the set of the functions where logarithmic means converges in measure is of first Baire category. From this result it follows that (Corollary 2.2) in classes wider than $L \log ^{+} L\left(I^{2}\right)$ there exists functions $f$ for which logarithmic means $\mathbf{t}_{n}^{\mathcal{k}}(f)$ of quadratical partial sums of double Walsh-Kaczmarz series diverge in measure.

Thus, in question of convergence in measure logarithmic means of quadratical partial sums of double Walsh-Kaczmarz series differ from the Marcinkiewicz means and are like the usual quadratical partial sums of double Walsh-Fourier series.

\section{Main Results}

The main results of this paper are presented in the following proposition.

Theorem 2.1. Let $L_{\Phi}\left(I^{2}\right)$ be an Orlicz space, such that

$$
L_{\Phi}\left(I^{2}\right) \nsubseteq L \log ^{+} L\left(I^{2}\right)
$$

Then the set of the functions in the Orlicz space $L_{\Phi}\left(I^{2}\right)$ with logarithmic means of quadratical partial sums of double Walsh-Kaczmarz series convergent in measure on $I^{2}$ is of first Baire category in $L_{\Phi}\left(I^{2}\right)$.

Corollary 2.2. Let $\varphi:[0, \infty[\rightarrow[0, \infty[$ be a nondecreasing function satisfying for $x \rightarrow+\infty$ the condition

$$
\varphi(x)=o(x \log x) .
$$


Then there exists a function $f \in L\left(I^{2}\right)$ such that

(a)

$$
\int_{I^{2}} \varphi(|f(x, y)|) d x d y<\infty
$$

(b) logarithmic means of quadratical partial sums of double Walsh-Kaczmarz series of $f$ diverge in measure on $I^{2}$.

\section{Auxiliary Results}

It is well-known $[2,3]$ for the Dirichlet kernel function that

$$
\left|D_{n}^{w}(x)\right|<\frac{1}{x}
$$

for any $0<x<1$. Then for these $x^{\prime}$ s we also get

$$
\left|F_{n}^{w}(x)\right|<\frac{1}{x}
$$

where $n \in \mathbf{N}$ is a nonnegative integer. It is also well-known for the Walsh-Paley-Dirichlet kernel functions the following lower pointwise estimates holds. Let $p_{A}=2^{2 A}+\cdots+2^{2}+2^{0}$ $(A \in \mathbf{N})$. Then for any $2^{-2 A-1} \leq x<1$ and $A \in \mathbf{N}$, we have

$$
\left|D_{p_{A}}^{w}(x)\right| \geq \frac{1}{4 x}
$$

Since this inequality plays a prominent role in the proofs of some divergence results concerning the partial sums of the Fourier series, then it seems that it would be useful to get a similar inequality also for the logarithmic kernels. In [20] the first author, Gát, and Tkebuchava proved the inequality

$$
\left|F_{p_{A}}^{w}(x)\right| \geq c \frac{\log (1 / x)}{x \log p_{A}}
$$

for all $1 \leq A \in \mathbf{N}$, and $x \in\left(2^{-2 A-1}, 1\right) \backslash J$. We have the exceptional set $J$, such that is "rare around zero." For $t=t_{0}, t_{0}+1, \ldots, 2 A, t_{0}=\inf \left\{t:\left\lfloor\left(l_{p_{[t / 2]-1}} / 16\right)-2^{15}\right\rfloor>1\right\}$ set $\tilde{t}:=$ $\left\lfloor\left(l_{p_{[t / 2]-1}} / 16\right)-2^{15}\right\rfloor$ (where $\lfloor u\rfloor$ denotes the lower integral part of $u$ ), and we take a "small part" of the interval $I_{t} \backslash I_{t+1}=\left[2^{-t-1}, 2^{-t}\right)$. This way the intervals are defined as follows:

$$
\Delta_{t}:=\left[\frac{1}{2^{t+1}}, \frac{1}{2^{t+1}}+\frac{1}{2^{t+\tilde{t}}}\right) .
$$

The exceptional set $J$ is given by

$$
J:=\bigcup_{t=t_{0}}^{\infty} \Delta_{t}
$$


For the logarithmic kernels $\mathbf{F}_{p_{A}}^{\kappa}$ of quadratical partial sums of double Walsh-KaczmarzFourier series, we prove an analogue result. To do this we need the following lemma of the first author, Gát and Tkebuchava [22].

Lemma 3.1 (see [22]). Let $s, t=0,1, \ldots, 2 A, s \leq t$, and $(x, y) \in\left(J_{s} \times J_{t}\right) \backslash\left(Q_{t+\tilde{s}}^{(s, t)} \cup\left(\Delta_{s} \times \Delta_{t}\right)\right)$. Then one has

$$
\left|\mathbf{F}_{p_{A}}^{w}(x, y)\right| \geq c \frac{2^{t+s} S}{\log p_{A}}
$$

where $J_{t}:=I_{t} \backslash I_{t+1}$ and

$$
\begin{aligned}
Q_{B}^{(s, t)}:= & \bigcup_{x_{s+1}+\cdots+x_{t-1} \leq 1} \bigcup_{x_{t}=0}^{1} \cdots \bigcup_{x_{B-1}=0}^{1} I_{B}\left(0, \ldots, 0,1, x_{s+1}, \ldots, x_{B-1}\right) \\
& \times I_{B}\left(0, \ldots, 0,1, y_{t+1}=x_{t+1}, \ldots, y_{B-1}=x_{B-1}\right) .
\end{aligned}
$$

Now, for the Walsh-Kaczmarz logarithmic kernels $\mathbf{F}_{p_{A}}^{\kappa}$, we prove the following.

Lemma 3.2. Let $x \in I_{2 A}\left(1, x_{1}, \ldots, x_{t-2}, x_{t-1}=1, x_{t}, \ldots, x_{s-1}, x_{s}=1, x_{s+1}=1,0, \ldots, 0\right)=: I_{2 A^{\prime}}^{t, s} y \in$ $I_{2 A}\left(1, y_{1}, \ldots, y_{t-2}, y_{t-1}=0, y_{t}=1, y_{t+1}=1,0, \ldots, 0\right)=: \tilde{I}_{2 A}^{t}$ for $s, t=2,3, \ldots, A$ and $t \leq s$. Then

$$
\left|\mathbf{F}_{p_{A}}^{\kappa}(x, y)\right| \geq c 2^{4 A-t-s}
$$

Proof. Set $(x, y) \in I_{2 A}^{t, s} \times \widetilde{I}_{2 A}^{t}$. Let

$$
\mathrm{G}_{p_{A}}^{\alpha}(x, y):=l_{p_{A}} \mathbf{F}_{p_{A}}^{\alpha}(x, y), \quad G_{p_{A}}^{\alpha}(x):=l_{p_{A}} F_{p_{A}}^{\alpha}(x)
$$

for $\alpha=w$ or $\kappa$. Now, we write that

$$
\mathbf{G}_{p_{A}}^{\kappa}(x, y)=\sum_{j=1}^{2^{2 A}} \frac{D_{j}^{\kappa}(x) D_{j}^{\kappa}(y)}{p_{A}-j}+\sum_{j=2^{2 A}+1}^{p_{A}-1} \frac{D_{j}^{\kappa}(x) D_{j}^{\kappa}(y)}{p_{A}-j}:=I+I I .
$$

First, we discuss II. We use equality (1.13)

$$
\begin{aligned}
I I= & \sum_{j=1}^{p_{A-1}-1} \frac{D_{j+2^{2 A}}^{\kappa}(x) D_{j+2^{2 A}}^{\kappa}(y)}{p_{A-1}-j} \\
= & l_{p_{A-1}} D_{2^{2 A}}(x) D_{2^{2 A}}(y)+D_{2^{2 A}}(x) r_{2 A}(y) G_{p_{A-1}}^{w}\left(\tau_{2 A}(y)\right) \\
& +D_{2^{2 A}}(y) r_{2 A}(x) G_{p_{A-1}}^{w}\left(\tau_{2 A}(x)\right)+r_{2 A}(x) r_{2 A}(y) G_{p_{A-1}}^{w}\left(\tau_{2 A}(x), \tau_{2 A}(y)\right) .
\end{aligned}
$$


If $(x, y) \in I_{2 A}^{t, s} \times \widetilde{I}_{2 A^{\prime}}^{t}$ then (see (1.14))

$$
\begin{gathered}
D_{2^{2 A}}(x)=D_{2^{2 A}}(y)=0, \\
\tau_{2 A}(x)=\left(0, \ldots, 0, x_{s+1}=1, x_{s}=1, x_{s-1}, \ldots, x_{t}, x_{t-1}=1, x_{t-2}, \ldots, x_{1}, x_{0}=1, x_{2 A}, \ldots\right), \\
\tau_{2 A}(y)=\left(0, \ldots, 0, y_{t+1}=1, y_{t}=1, y_{t-1}=0, y_{t-2}, \ldots, y_{1}, y_{0}=1, y_{2 A}, \ldots\right) .
\end{gathered}
$$

Moreover, by Lemma 3.1, we have

$$
|I I|=\left|\mathrm{G}_{p_{A-1}}^{w}\left(\tau_{2 A}(x), \tau_{2 A}(y)\right)\right| \geq c(2 A-s) 2^{4 A-t-s} .
$$

Now, we discuss $I$ by the help of (1.13)

$$
\begin{aligned}
I= & \sum_{l=0}^{2 A-1} \sum_{j=2^{l}}^{2^{l+1}-1} \frac{D_{j}^{\kappa}(x) D_{j}^{\kappa}(y)}{p_{A}-j}+\frac{D_{2^{2 A}}(x) D_{2^{2 A}}(y)}{p_{A-1}} \\
= & \sum_{l=0}^{2 A-1} \sum_{j=0}^{2^{l}-1} \frac{D_{j+2^{l}}^{\kappa}(x) D_{j+2^{l}}^{\kappa}(y)}{p_{A}-j-2^{l}} \\
= & \sum_{l=0}^{2 A-1} \sum_{j=0}^{2^{l}-1} \frac{D_{2^{l}}(x) D_{2^{l}}(y)}{p_{A}-j-2^{l}}+\sum_{l=0}^{2 A-1} D_{2^{l}}(x) \sum_{j=0}^{2^{l}-1} \frac{r_{l}(y) D_{j}^{w}\left(\tau_{l}(y)\right)}{p_{A}-j-2^{l}} \\
& +\sum_{l=0}^{2 A-1} D_{2^{l}}(y) \sum_{j=0}^{2^{l}-1} \frac{r_{l}(x) D_{j}^{w}\left(\tau_{l}(x)\right)}{p_{A}-j-2^{l}}+\sum_{l=0}^{2 A-1} \sum_{j=0}^{2^{l}-1} \frac{r_{l}(x) r_{l}(y) D_{j}^{w}\left(\tau_{l}(x)\right) D_{j}^{w}\left(\tau_{l}(y)\right)}{p_{A}-j-2^{l}} .
\end{aligned}
$$

Since $x_{0}=y_{0}=1, D_{2^{l}}(x)=D_{2^{l}}(y)=0$ for all $l \geq 1$, thus,

$$
\begin{aligned}
I & =\frac{1}{p_{A}-1}+\sum_{l=1}^{2 A-1} r_{l}(x) r_{l}(y) \sum_{j=1}^{2^{l}-1} \frac{D_{j}^{w}\left(\tau_{l}(x)\right) D_{j}^{w}\left(\tau_{l}(y)\right)}{p_{A}-j-2^{l}} \\
& =: \frac{1}{p_{A}-1}+\sum_{l=1}^{2 A-1} I_{l} .
\end{aligned}
$$

We use Abel's transformation for $I_{l}(l \geq 1)$

$$
\begin{aligned}
I_{l}= & r_{l}(x) r_{l}(y) \sum_{j=1}^{2^{l}-2}\left(\frac{1}{p_{A}-j-2^{l}}-\frac{1}{p_{A}-j-2^{l}-1}\right) j \mathcal{K}_{j}^{w}\left(\tau_{l}(x), \tau_{l}(y)\right) \\
& +r_{l}(x) r_{l}(y) \frac{\left(2^{l}-1\right) \mathcal{K}_{2^{l}-1}^{w}\left(\tau_{l}(x), \tau_{l}(y)\right)}{p_{A}-2^{l+1}+1}=: I_{l}^{1}+I_{l}^{2} .
\end{aligned}
$$


We write

$$
\begin{gathered}
\left|I_{l}^{1}\right| \leq \frac{c}{2^{4 A}} \sum_{j=1}^{2^{l}-2} j\left|\mathcal{K}_{j}^{w}\left(\tau_{l}(x), \tau_{l}(y)\right)\right| \\
\left|I_{l}^{2}\right| \leq \frac{c}{2^{2 A}}\left(2^{l}-1\right)\left|\mathcal{K}_{2^{l}-1}^{w}\left(\tau_{l}(x), \tau_{l}(y)\right)\right| .
\end{gathered}
$$

Since $\left|D_{i}\right| \leq i$, we have

$$
n\left|\mathcal{K}_{n}^{w}\right| \leq\left|\sum_{i=0}^{n} D_{i}^{w} D_{i}^{w}\right| \leq c n^{3} .
$$

For $l \leq s+2$, we obtain that

$$
\left|I_{l}^{1}\right| \leq c \frac{2^{4 l}}{2^{4 A}}, \quad\left|I_{l}^{2}\right| \leq c \frac{2^{3 l}}{2^{2 A}}
$$

Moreover,

$$
\begin{gathered}
\sum_{l=1}^{s+2}\left|I_{l}^{1}\right| \leq \frac{c}{2^{4 A}} \sum_{l=1}^{s+2} 2^{4 l} \leq c \frac{2^{4 s}}{2^{4 A}}<c \\
\sum_{l=1}^{s+2}\left|I_{l}^{2}\right| \leq \frac{c}{2^{2 A}} \sum_{l=1}^{s+2} 2^{3 l} \leq \frac{c 2^{3 s}}{2^{2 A}} \leq c 2^{A} .
\end{gathered}
$$

Let $s+2<l<2 A$. Then we have

$$
\begin{gathered}
\tau_{l}(x)=\left(0, \ldots, 0,1,1, x_{s-1}, \ldots, x_{t-1}=1, \ldots, x_{1}, 1,0, \ldots, 0, x_{2 A}, \ldots\right), \\
\tau_{l}(y)=\left(0, \ldots, 0,1,1, y_{t-1}=0, y_{t-2}, \ldots, y_{1}, 1,0, \ldots, 0, y_{2 A}, \ldots\right),
\end{gathered}
$$

that is, $\tau_{l}(x) \in I_{l-s-3} \backslash I_{l-s-2}, \tau_{l}(y) \in I_{l-t-3} \backslash I_{l-t-2}$.

Now, we introduce the following notation:

$$
\mathcal{K}_{a, b}^{w}(x, y):=\sum_{j=a}^{a+b-1} D_{j}^{w}(x) D_{j}^{w}(y)
$$

and $n^{(s)}:=\sum_{i=s}^{\infty} n_{i} 2^{i}(n, s \in \mathbf{N})$. A simple consideration gives

$$
n \mathcal{K}_{n}^{w}(x, y)=\sum_{s=0}^{|n|} n_{s} \mathcal{K}_{n^{(s+1)}, 2^{s}}(x, y)+D_{n}^{w}(x) D_{n}^{w}(y) .
$$


It is known [23] that if $t \leq k$ and $(x, y) \in\left(I_{t} \backslash I_{t+1}\right) \times\left(I_{k} \backslash I_{k+1}\right)$, then

$$
\left|\mathcal{K}_{n^{(s+1)}, 2^{s}}^{w}(x, y)\right| \leq c 2^{s+t+k}
$$

Using this inequality, we have that

$$
\begin{aligned}
\left|I_{l}^{1}\right| & \leq \frac{c}{2^{4 A}} \sum_{k=0}^{l-1} \sum_{j=2^{k}}^{2^{k+1}-1} j\left|\mathcal{K}_{j}^{w}\left(\tau_{l}(x), \tau_{l}(y)\right)\right| \\
& \leq \frac{c}{2^{4 A}} \sum_{k=0}^{l-1} \sum_{j=2^{k}}^{2^{k+1}-1}\left(\sum_{v=0}^{k}\left|\mathcal{K}_{j^{(v+1)}, 2^{v}}^{w}\left(\tau_{l}(x), \tau_{l}(y)\right)\right|+\left|D_{j}\left(\tau_{l}(x)\right) D_{j}\left(\tau_{l}(y)\right)\right|\right) \\
& \leq \frac{c}{2^{4 A}} \sum_{k=0}^{l-1} \sum_{j=2^{k}}^{2^{k+1}-1}\left(\sum_{v=0}^{k} 2^{v+2 l-t-s}+j^{2}\right) \\
& \leq c \frac{2^{4 l-t-s}}{2^{4 A}}+c \frac{2^{3 l}}{2^{4 A}} .
\end{aligned}
$$

Moreover,

$$
\sum_{l=s+3}^{2 A-1}\left|I_{l}^{1}\right| \leq c \frac{2^{8 A-t-s}}{2^{4 A}}+c \frac{2^{6 A}}{2^{4 A}} \leq c 2^{4 A-t-s}
$$

Analogously,

$$
\begin{gathered}
\left|I_{l}^{2}\right| \leq \frac{c}{2^{2 A}}\left(\sum_{v=0}^{l-1} 2^{v+2 l-t-s}+2^{2 l}\right) \leq c \frac{2^{3 l-t-s}}{2^{2 A}}+c \frac{2^{2 l}}{2^{2 A}} \\
\sum_{l=s+3}^{2 A-1}\left|I_{l}^{2}\right| \leq c 2^{4 A-t-s} .
\end{gathered}
$$

Taking into account (3.11), we get

$$
\left|\mathbf{F}_{p_{A}}^{\kappa}(x, y)\right| \geq \frac{|I I|-|I|}{l_{p_{A}}} \geq c_{1} \frac{|I I|-|I|}{2 A} .
$$

Therefore by (3.14)-(3.29), it follows that

$$
\left|\mathbf{F}_{p_{A}}^{\kappa}(x, y)\right| \geq c \cdot c_{1}\left(1-\frac{s}{2 A}\right) 2^{4 A-t-s}-\frac{\tilde{c}}{2 A} 2^{4 A-t-s}
$$

with suitable constants $c, c_{1}, \widetilde{c}>0$. It is clear that for $A$ large enough we have

$$
\left|\mathbf{F}_{p_{A}}^{\kappa}(x, y)\right| \geq c_{*} 2^{4 A-t-s}
$$

where $c_{*}>0$ is an absolute constant. 
Journal of Function Spaces and Applications

We apply the reasoning of [24] formulated as the following proposition in particular case.

Theorem A. Let $H: L^{1}\left(I^{2}\right) \rightarrow L^{0}\left(I^{2}\right)$ be a linear continuous operator, which commutes with family of translations $\mathcal{E}$, that is, for all $E \in \mathcal{E}$ for all $f \in L^{1}\left(I^{2}\right) H E f=E H f$. Let $\|f\|_{L^{1}\left(I^{2}\right)}=1$ and $\lambda>1$. Then for any $1 \leq r \in \mathbb{N}$ under condition, $\operatorname{mes}\left\{(x, y) \in I^{2}:|H f|>\lambda\right\} \geq 1 / r$, there exist $E_{1}, \ldots, E_{r}, E_{1}^{\prime}, \ldots, E_{r}^{\prime} \in \mathcal{E}$ and $\varepsilon_{i}= \pm 1, i=1, \ldots, r$, such that

$$
\operatorname{mes}\left\{(x, y) \in I^{2}:\left|H\left(\sum_{i=1}^{r} \varepsilon_{i} f\left(E_{i} x, E_{i}^{\prime} y\right)\right)\right|>\lambda\right\} \geq \frac{1}{8}
$$

Theorem B. Let $\left\{H_{m}\right\}_{m=1}^{\infty}$ be a sequence of linear continuous operators, acting from Orlicz space $L_{\Phi}\left(I^{2}\right)$ into the space $L^{0}\left(I^{2}\right)$. Suppose that there exist the sequence of functions $\left\{\xi_{k}\right\}_{k=1}^{\infty}$ from unit bull $S_{\Phi}(0,1)$ of space $L_{\Phi}\left(I^{2}\right)$, and sequences of integers $\left\{m_{k}\right\}_{k=1}^{\infty}$ and $\left\{v_{k}\right\}_{k=1}^{\infty}$ increasing to infinity such that

$$
\varepsilon_{0}=\inf _{k} \operatorname{mes}\left\{(x, y) \in I^{2}:\left|H_{m_{k}} \xi_{k}(x, y)\right|>v_{k}\right\}>0 .
$$

Then $B$-the set of functions $f$ from space $L_{\Phi}\left(I^{2}\right)$, for which the sequence $\left\{H_{m} f\right\}$ converges in measure to an a.e. finite function - is of first Baire category in space $L_{\Phi}\left(I^{2}\right)$.

Theorem GGT. Let $L_{\Phi}$ be an Orlicz space, and let $\varphi:[0, \infty) \rightarrow[0, \infty)$ be measurable function with condition $\varphi(x)=o(\Phi(x))$ as $x \rightarrow \infty$. Then there exists Orlicz space $L_{\omega}$, such that $\omega(x)=$ $o(\Phi(x))$ as $x \rightarrow \infty$, and $\omega(x) \geq \varphi(x)$ for $x \geq c \geq 0$.

The proof of Theorems B and GGT can be found in [20].

\section{Proof of the Theorem}

Proof of Theorem 2.1. By Theorem B the proof of Theorem 2.1 will be complete if we show that there exist sequences of integers $\left\{A_{k}: k \geq 1\right\}$ and $\left\{v_{k}: k \geq 1\right\}$ increasing to infinity, and a sequence of functions $\left\{\xi_{k}: k \geq 1\right\}$ from the unit bull $S_{\Phi}(0,1)$ of Orlicz space $L_{\Phi}\left(I^{2}\right)$, such that for all $k$

$$
\operatorname{mes}\left\{(x, y) \in I^{2}:\left|\mathbf{t}_{p_{A_{k}}}^{\kappa}\left(\xi_{k}, x, y\right)\right|>v_{k}\right\} \geq \frac{1}{8}
$$

First, we prove that there exists $c>0$ such that

$$
\operatorname{mes}\left\{(x, y) \in I^{2}:\left|\mathbf{t}_{p_{A_{k}}^{\kappa}}^{\kappa}\left(D_{2^{2 A+1}} \otimes D_{2^{2 A+1}} ; x, y\right)\right|>c 2^{3 A}\right\}>c \frac{A}{2^{3 A}} \text {. }
$$

It is easy to show that

$$
\mathbf{t}_{p_{A_{k}}}^{\kappa}\left(D_{2^{2 A+1}} \otimes D_{2^{2 A+1}} ; x, y\right)=S_{2^{2 A+1}, 2^{2 A+1}}\left(\mathbf{F}_{p_{A}}^{\kappa} ; x, y\right)=\mathbf{F}_{p_{A}}^{\kappa}(x, y)
$$


Hence, from Lemma 3.2, we can write

$$
\left|\mathbf{t}_{p_{A_{k}}}^{\kappa}\left(D_{2^{2 A+1}} \otimes D_{2^{2 A+1}} ; x, y\right)\right|=\left|\mathbf{F}_{p_{A}}^{\kappa}(x, y)\right| \geq c 2^{4 A-t-s},
$$

for $(x, y) \in I_{2 A}^{t, s} \times \widetilde{I}_{2 A}^{t}$.

Since

$$
c 2^{4 A-t-s}>c 2^{3 A}
$$

for $t+s<A$ and $0 \leq t \leq s<A$, from (4.4) we conclude that

$$
\begin{aligned}
\operatorname{mes}\{(x, y) & \left.\in I^{2}:\left|\mathbf{t}_{p_{A}}^{\kappa}\left(D_{2^{2 A+1}} \otimes D_{2^{2 A+1}} ; x, y\right)\right|>c 2^{3 A}\right\} \\
& \geq c \sum_{s=[A / 2]}^{A} \sum_{t=0}^{A-s} \operatorname{mes}\left(I_{2 A}^{t, s} \times \widetilde{I}_{2 A}^{t}\right) \\
& \geq c \sum_{s=[A / 2]}^{A} \sum_{t=0}^{A-s} \frac{2^{s}}{2^{2 A}} \frac{2^{t}}{2^{2 A}} \\
& \geq c \sum_{s=[A / 2]}^{A} \frac{2^{s}}{2^{2 A}} \frac{2^{A-s}}{2^{2 A}} \\
& \geq \frac{c A}{2^{3 A}} .
\end{aligned}
$$

Hence, (4.2) is proved.

From the condition of the theorem, we write [1]

$$
\liminf _{u \rightarrow \infty} \frac{\Phi(u)}{u \log u}=0
$$

Consequently, there exists a sequence of integers $\left\{A_{k}\right\}_{k=1}^{\infty}$ increasing to infinity, such that

$$
\lim _{k \rightarrow \infty} \Phi\left(2^{4 A_{k}}\right) 2^{-4 A_{k}} A_{k}^{-1}=0, \quad \frac{\Phi\left(2^{4 A_{k}}\right)}{2^{4 A_{k}}} \geq 4, \forall k
$$

From (4.2) we write

$$
\operatorname{mes}\left\{(x, y) \in I^{2}:\left|\mathbf{t}_{p_{A_{k}}}^{\kappa}\left(D_{2^{2 A_{k}+1}} \otimes D_{2^{2 A_{k}+1}} ; x, y\right)\right|>c 2^{3 A_{k}}\right\}>c \frac{A_{k}}{2^{3 A_{k}}}
$$


Then by the virtue of Theorem A, there exists $e_{1}, \ldots, e_{r}, e_{1}^{\prime}, \ldots, e_{r}^{\prime} \in[0,1]$ and $\varepsilon_{1}, \ldots, \varepsilon_{r}= \pm 1$ such that

$$
\operatorname{mes}\left\{(x, y) \in I^{2}:\left|\sum_{i=1}^{r} \varepsilon_{i} \mathbf{t}_{p_{A_{k}}}^{\kappa}\left(D_{2^{2 A_{k}+1}} \otimes D_{2^{2 A_{k}+1}}, e_{i} \oplus x, e_{i}^{\prime} \oplus y\right)\right|>2^{3 A_{k}}\right\}>\frac{1}{8},
$$

where $r=\left[2^{3 A_{k}} /\left(c A_{k}\right)\right]+1$.

Set

$$
\xi_{k}(x, y)=\frac{2^{4 A_{k}-1}}{\Phi\left(2^{4 A_{k}}\right)} M_{k}(x, y)
$$

where

$$
M_{k}(x, y)=\frac{1}{r} \sum_{i=1}^{r} \varepsilon_{i} D_{2^{2 A_{k}+1}}\left(e_{i} \oplus x\right) D_{2^{2 A_{k}+1}}\left(e_{i}^{\prime} \oplus y\right) .
$$

\section{Denote}

$$
v_{k}=\frac{2^{7 A_{k}-1}}{r \Phi\left(2^{4 A_{k}}\right)}
$$

It is easy to show that

$$
\begin{aligned}
& \operatorname{mes}\left\{(x, y) \in I^{2}:\left|\mathbf{t}_{p_{A_{k}}}^{\kappa}\left(\xi_{k}, x, y\right)\right|>v_{k}\right\} \\
& =\operatorname{mes}\left\{(x, y) \in I^{2}:\left|\sum_{i=1}^{r} \varepsilon_{i} \mathbf{t}_{p_{A_{k}}}^{\kappa}\left(D_{2^{2 A_{k}+1}} \otimes D_{2^{2 A_{k}+1}}, e_{i} \oplus x, e_{i}^{\prime} \oplus y\right)\right|>2^{3 A_{k}}\right\} \\
& >\frac{1}{8}
\end{aligned}
$$

Hence, (4.1) is proved.

Since

$$
\left\|M_{k}\right\|_{L^{\infty}\left(I^{2}\right)} \leq 2^{4 m_{k}+2}, \quad\left\|M_{k}\right\|_{L^{1}\left(I^{2}\right)} \leq 1 .
$$

Moreover,

$$
\begin{aligned}
& \left|\int_{I^{2}} f g\right| \leq \frac{1}{2}\left[\int_{I^{2}} \Phi(2|f|)+1\right], \\
& \frac{\Phi(u)}{u}<\frac{\Phi\left(u^{\prime}\right)}{u^{\prime}} \quad\left(0<u<u^{\prime}\right) .
\end{aligned}
$$


From (4.8) we can write

$$
\begin{aligned}
\left\|\xi_{k}\right\|_{L_{\Phi}\left(I^{2}\right)} & \leq \frac{1}{2}\left[1+\int_{I^{2}} \Phi\left(\frac{2^{4 A_{k}}\left|M_{k}(x, y)\right|}{\Phi\left(2^{4 A_{k}}\right)}\right) d x d y\right] \\
& \leq \frac{1}{2}\left[1+\int_{I^{2}} \frac{\Phi\left(2^{4 A_{k}} 2^{4 A_{k}+2} /\left(\Phi\left(2^{4 A_{k}}\right)\right)\right)}{2^{4 A_{k}}\left(2^{4 A_{k}+2} /\left(\Phi\left(2^{4 A_{k}}\right)\right)\right)} \frac{2^{4 A_{k}}\left|M_{k}(x, y)\right|}{\Phi\left(2^{4 A_{k}}\right)} d x d y\right] \\
& \leq \frac{1}{2}\left[1+\int_{I^{2}} \frac{\Phi\left(2^{4 A_{k}}\right)}{2^{4 A_{k}}} \frac{2^{4 A_{k}}\left|M_{k}(x, y)\right|}{\Phi\left(2^{4 A_{k}}\right)} d x d y\right]
\end{aligned}
$$$$
\leq 1 \text {. }
$$

Hence, $\xi_{k} \in S_{\Phi}(0,1)$, and Theorem 2.1 is proved.

The validity of Corollary 2.2 follows immediately from Theorems 2.1 and GGT.

\section{References}

[1] M. A. Krasnosel'skii and Y. B. Rutickii, Convex Functions and Orlicz Space, P. Noorhoff, Groningen, The Netherlands, 1961.

[2] B. I. Golubov, A. V. Efimov, and V. A. Skvorcov, Series and Transformations of Walsh, Nauka, Moscow, Russia, 1987.

[3] F. Schipp, W. R. Wade, and P. Simon, Walsh Series, Introduction to Dyadic Harmonic Analysis, Adam Hilger Ltd, Bristol, UK, 1990.

[4] V. A. Skvorcov, "On Fourier series with respect to the Walsh-Kaczmarz system," Analysis Mathematica, vol. 7, no. 2, pp. 141-150, 1981.

[5] A. A. Šneǐder, “On series of Walsh functions with monotonic coefficients," Izvestiya Akademii Nauk SSSR, vol. 12, pp. 179-192, 1948.

[6] L. A. Balašov, "Series with respect to the Walsh system with monotone coefficients," Sibirskiu Matematičeskĭ Žurnal, vol. 12, pp. 25-39, 1971 (Russian).

[7] F. Schipp, "Pointwise convergence of expansions with respect to certain product systems," Analysis Mathematica, vol. 2, no. 1, pp. 65-76, 1976.

[8] W. S. Young, "On the a.e. convergence of Walsh-Kaczmarz-Fourier series," Proceedings of the American Mathematical Society, vol. 44, pp. 353-358, 1974.

[9] G. Gát, "On $(C, 1)$ summability of integrable functions with respect to the Walsh-Kaczmarz system," Studia Mathematica, vol. 130, no. 2, pp. 135-148, 1998.

[10] P. Simon, "On the Cesaro summability with respect to the Walsh-Kaczmarz system," Journal of Approximation Theory, vol. 106, no. 2, pp. 249-261, 2000.

[11] P. Simon, " $(C, \alpha)$ summability of Walsh-Kaczmarz-Fourier series," Journal of Approximation Theory, vol. 127, no. 1, pp. 39-60, 2004.

[12] K. Nagy, "On the two-dimensional Marcinkiewicz means with respect to Walsh-Kaczmarz system," Journal of Approximation Theory, vol. 142, no. 2, pp. 138-165, 2006.

[13] L. V. Zhizhiashvili, "Some problems of multidimensional harmonic analysis," TGU, Tbilisi, 1996.

[14] R. Getsadze, "On the divergence in measure of multiple Fourier seties," Some Problems of Functions Theory, vol. 4, pp. 84-117, 1988 (Russian).

[15] S. V. Konyagin, "On a subsequence of Fourier-Walsh partial sums," Rossiǔskaya Akademiya Nauk, vol. 54 , no. 4, pp. 69-75, 1993.

[16] G. Tkebuchava, "Subsequences of partial sums of multiple Fourier and Fourier-Walsh series," Bulletin of the Georgian Academy of Sciences, vol. 169, no. 2, pp. 252-253, 2004.

[17] U. Goginava, "Convergence in measure of partial sums of double Fourier series with respect to the Walsh-Kaczmarz system," Journal of Mathematical Analysis and Approximation Theory, vol. 2, no. 2, pp. 160-167, 2007. 
[18] U. Goginava and K. Nagy, "Weak type inequality for logarithmic means of Walsh-Kaczmarz-Fourier series," Real Analysis Exchange, vol. 35, no. 2, pp. 445-461, 2010.

[19] G. Gát and U. Goginava, "On the divergence of Nörlund logarithmic means of Walsh-Fourier series," Acta Mathematica Sinica, vol. 25, no. 6, pp. 903-916, 2009.

[20] G. Gát, U. Goginava, and G. Tkebuchava, "Convergence in measure of logarithmic means of double Walsh-Fourier series," Georgian Mathematical Journal, vol. 12, no. 4, pp. 607-618, 2005.

[21] G. Gát, U. Goginava, and G. Tkebuchava, "Convergence of logarithmic means of multiple WalshFourier series," Analysis in Theory and Applications, vol. 21, no. 4, pp. 326-338, 2005.

[22] G. Gát, U. Goginava, and G. Tkebuchava, "Convergence in measure of logarithmic means of quadratical partial sums of double Walsh-Fourier series," Journal of Mathematical Analysis and Applications, vol. 323, no. 1, pp. 535-549, 2006.

[23] K. Nagy, "Some convergence properties of the Walsh-Kaczmarz system with respect to the Marcinkiewicz means," Rendiconti del Circolo Matematico di Palermo. Serie II, no. 76, pp. 503-516, 2005.

[24] A. M. Garsia, Topics in Almost Everywhere Convergence, vol. 4, Markham Publishing Co., Chicago, Ill, USA, 1970. 


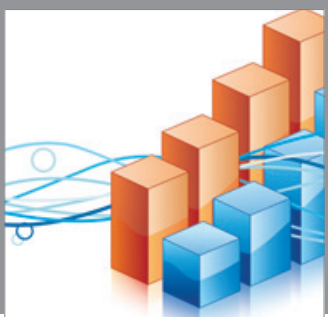

Advances in

Operations Research

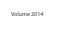

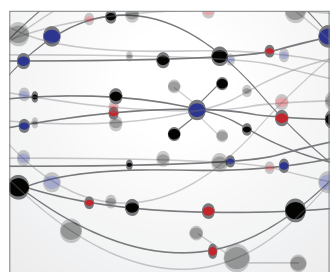

\section{The Scientific} World Journal
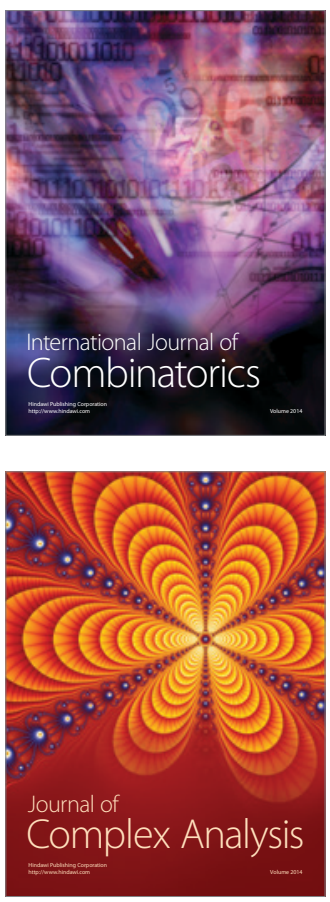

International Journal of

Mathematics and

Mathematical

Sciences
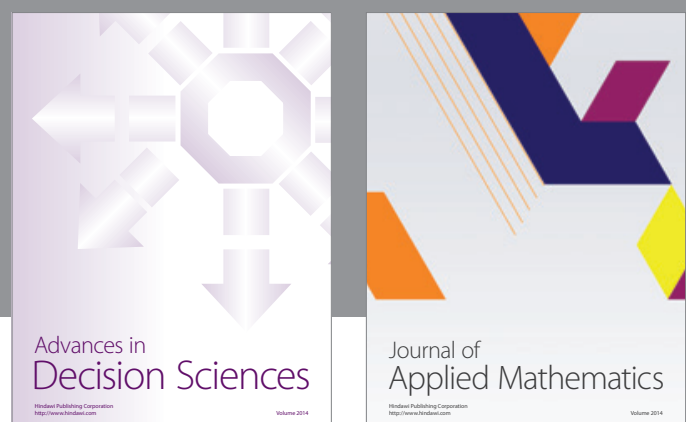

Journal of

Applied Mathematics
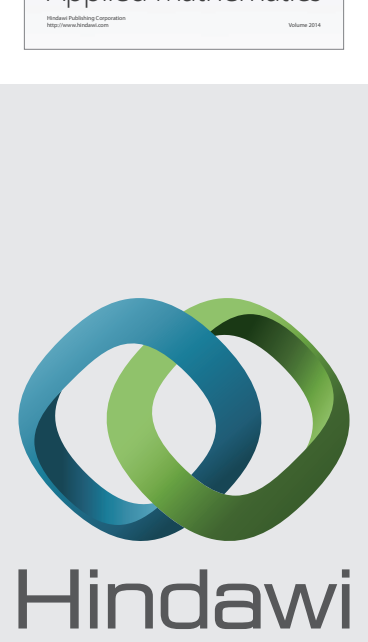

Submit your manuscripts at http://www.hindawi.com
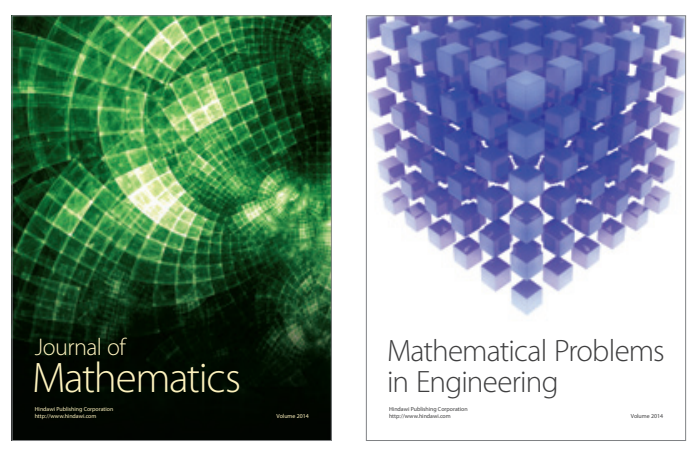

Mathematical Problems in Engineering
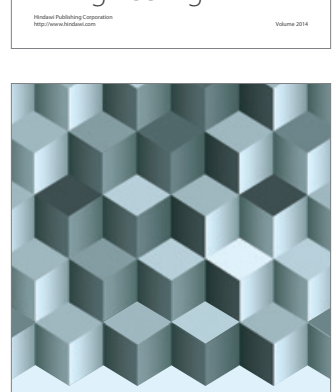

Journal of

Function Spaces
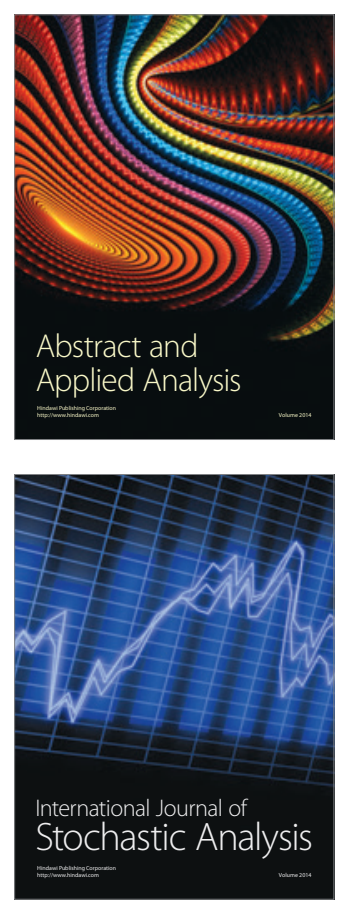

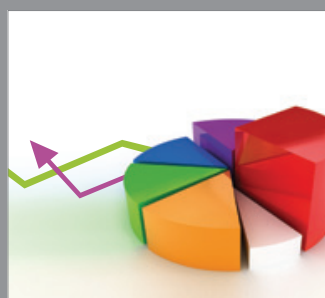

ournal of

Probability and Statistics

Promensencen
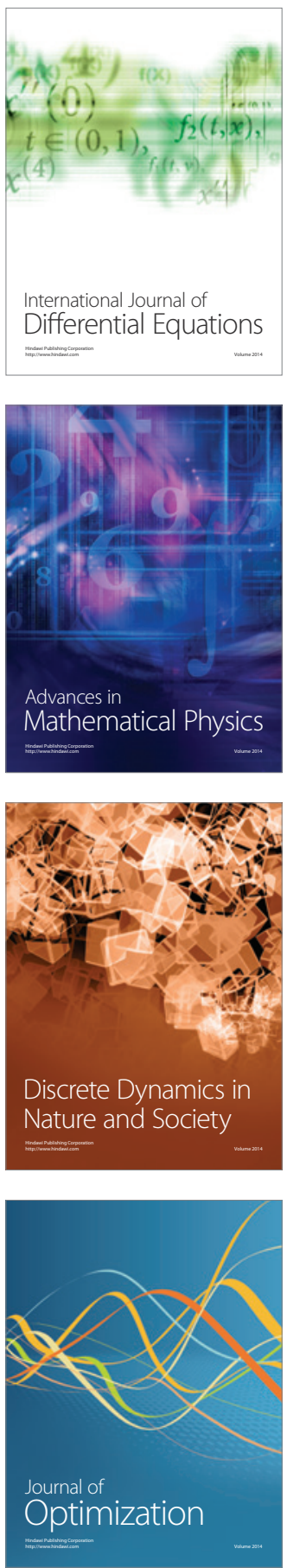